\title{
Component mode synthesis using undeformed interface coupling modes to connect soft and stiff substructures
}

\author{
Eskil Lindberg*, Nils-Erik Hörlin and Peter Göransson \\ KTH Royal Institute of Technology, Department of Aeronautical and Vehicle Engineering, MWL the Marcus \\ Wallenberg Laboratory for sound and vibration research, Stockholm Sweden
}

Received 5 December 2011

Revised 27 April 2012

\begin{abstract}
Classical component mode synthesis methods for reduction are usually limited by the size and compatibility of the coupling interfaces. A component mode synthesis approach with constrained coupling interfaces is presented for vibro-acoustic modelling. The coupling interfaces are constrained to six displacement degrees of freedom. These degrees of freedom represent rigid interface translations and rotations respectively, retaining an undeformed interface shape. This formulation is proposed for structures with coupling between softer and stiffer substructures in which the displacement is chiefly governed by the stiffer substructure. Such may be the case for the rubber-bushing/linking arm assembly in a vehicle suspension system. The presented approach has the potential to significantly reduce the modelling size of such structures, compared with classical component mode synthesis which would be limited by the modelling size of the interfaces. The approach also eliminates problems of nonconforming meshes in the interfaces since only translation directions, rotation axes and the rotation point need to be common for the coupled substructures. Simulation results show that the approach can be used for modelling of systems that resemble a vehicle suspension. It is shown for a test case that adequate engineering accuracy can be achieved when the stiffness properties of the connecting parts are within the expected range of rubber connected to steel.
\end{abstract}

Keywords: Vibro-acoustic, component mode synthesis, substructuring, vehicle suspension, rubber bushings

\section{Introduction}

Developing a vehicle design is a multidisciplinary process, in which a broad range of requirements must be satisfied. This may lead to compromises, or even to conflicting outcomes. For instance, it may be hard to satisfy the requirements both for greenhouse gas emissions and acoustics emissions. In general, an increased overall vehicle mass may help to reduce the interior noise emissions, but it might increase the greenhouse gas emissions. This and other similar conflicts may benefit from appropriate engineering solutions.

Such design solutions require a fundamental understanding of the physical properties involved. One area where there is a gap in this understanding is the vibro-acoustic behaviour of the suspension system. Vehicle manufacturers often try to overcome this gap using measurement techniques and signal processing to guide engineering experience.

Traditionally, the problems of the vibro-acoustic transfer path (or structural-borne noise) in the vehicle industry have been dealt with using correlation methods such as the multi-correlation function [1]. The relative contribution of multiple dynamic excitations is estimated by the coherence function [2]. Another commonly used method is

\footnotetext{
${ }^{*}$ Corresponding author: Eskil Lindberg, KTH Royal Institute of Technology, Department of Aeronautical and Vehicle Engineering, MWL the Marcus Wallenberg Laboratory for sound and vibration research, Stockholm Sweden. Tel.: +46 87 907610; E-mail: eskill@kth.se.
} 
experimental transfer path analysis (TPA). This method is considered to be one of the most important tools for analysing transfer paths in complex structures such as the suspension system of a vehicle [3].

Mathematical models can often help the understanding of the governing aspects of the physics. Mathematical modelling of the suspension system usually resorts to finite element methods (FEM), where simplifications of geometry and material properties may lead to substantial errors. In addition, simplified models in use today often do not model frequencies above around $200 \mathrm{~Hz}$ (e.g. see Kido et al. [4]). Detailed vibro-acoustic modelling of the vehicle suspension is a non-trivial task, because of geometrical complexity and the numerous connected parts of fundamentally different behaviour, such as the rubber bushings that are used to create a soft connection between stiffer parts. The geometrical complexity often leads to a very fine FE mesh merely to resolve the geometrical description of the parts, and the resulting number of degrees of freedom (DOFs) places high demands on computer resources to evaluate even single components. The fine mesh is often not necessary for the description of the vibroacoustic phenomena as such. To overcome this problem of the uncontrollable size of the coupled problem, methods to reduce the modelling size would be a good tool to utilise before the assembly of the complete problem.

In this paper, a reduction technique for modelling suspension systems is suggested. This technique offers the potential of overcoming the problem of an unnecessarily large number of DOFs in the mathematical description of the vibro-acoustic field.

Reduction methods in structural dynamics (vibro-acoustics) often use the approach of modes where a set of normal or "eigen" modes are generated from an eigenvalue problem. From the theory of modal superposition, it is known that a reduced set eigenmodes can be used to provide an approximate solution of the original problem. The choice of the set of modes included in the approximated solution may come from a physical motivation. The normal choice is only to include modes with a corresponding eigenfrequency below a particular frequency limit. The theory of how reduced subsystems can be coupled together is commonly referred to as component mode synthesis (CMS). CMS can be described as a method in which the local behaviour of single substructures is described by a set of reduced local eigenmodes, and force and displacement continuity between the substructures are enforced by a set of coupling functions (constraint modes).

A common CMS method was proposed by Craig and Bampton [5], but there are several different versions of the same idea, the main distinction of the different formulations being the use of fixed interface [5] or free interfaces ([6] or hybrid [7]). Common to all is that continuity between substructures is usually ensured by a static (or Guyan) condensation, where the static solution of the inner DOFs is computed. For a more in-depth description of the different formulations, the reader is referred to the review paper of De Klerk et al. [8]. A limitation of classical CMS methods such as the Craig-Bampton (C\&B) method is that the reduction order is limited by the size of the coupling interface, hence only appropriate for systems with small coupling interfaces [9-11]. The problem with non-conforming mesh interfaces is usually dealt with by introducing additional constraints in the form of what are known as Lagrange multipliers (see for instance $[12,13]$ ).

Research on the interface reduction is a research area which has a great potential and much work has been published on the subject, for instance, $[10,11,14,15]$. Most of the methods stem from the fact that a static (or Guyan) reduced system is generated by only using the static modes in the change of base. Thus, a new system only comprising of interface DOFs, can be used to generate yet another set of basis functions by solving the new eigenvalue problem. However, the physically motivated criteria of the eigenfrequency cannot be used to choose a reduced set of basis functions. Instead, the method is limited to criteria such as the evaluation of the energy strain of each modes [16] or singular value decomposition [15,17].

In this paper, a physically motivated technique of interface reduction is presented. The technique is proposed for coupling between soft and stiff parts, such as the connection to rubber bushings in the vehicle suspension system. The physical reasoning is based on the assumption that an interface between a soft and a stiff part will have an approximately undeformed interface. Hence, the displacement of the interface can be described by the six rigid "body" motions (three orthogonal spatial directions and the three rotations around these axes) [18-20].

This formulation also allows for a substantial reduction of the original problem and completely eliminates the problem of non-conforming meshes, since only a conforming coordinate system is needed when generating the undeformed interface displacement functions.

In Section 2 the general form of the classical C\&B CMS is presented together with a procedure to generate the undeformed displacement function used herein. In Section 3 the proposed approach is evaluated for a test case, and the results are compared to the direct solution and the classical C\&B method. Results and discussion around the industrial application of the approach are also included in this section. 


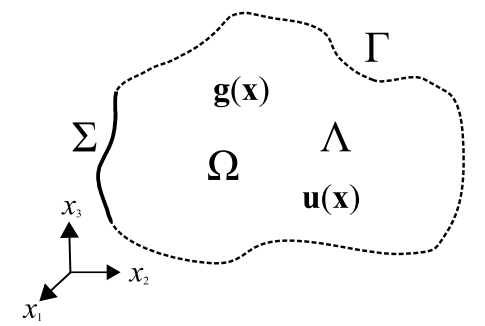

Fig. 1. The vibro-acoustic displacement $\mathbf{u}(\mathbf{x})$ and the body force $\mathbf{g}(\mathbf{x})$ in the domain $\Omega$ where $\Sigma=\partial \Omega \backslash \Gamma$, and $\Lambda=\Omega \cup \Gamma$. $\mathbf{x}=\left[x_{1} ; x_{2} ; x_{3}\right]$ is the position in space.

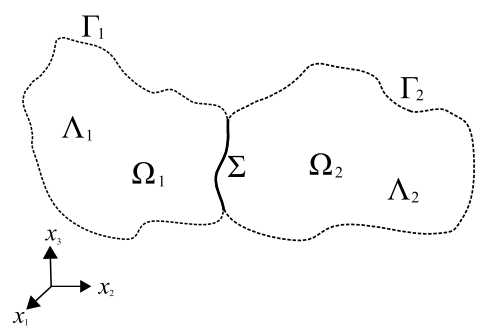

\section{Theory}

In this section the CMS technique used herein is presented. The method uses the classical C\&B formulation, where a slightly more general form is presented than traditionally given in the literature. The section ends with the formulation of the six undeformed coupling modes.

\subsection{General problem}

The governing equation of the vibro-acoustic system in Fig. 1 may be written as

$$
\nabla \cdot \boldsymbol{\sigma}(\mathbf{x})+\omega^{2} \rho(\mathbf{x}) \mathbf{u}(\mathbf{x})=\mathbf{g}(\mathbf{x}), \quad \forall \mathbf{x} \in \Omega
$$

$\rho(\mathbf{x})$ is the mass density field, $\boldsymbol{\sigma}(\mathbf{x})$ is the symmetric Cauchy stress matrix and $\mathbf{g}(\mathbf{x})$ is the given body force field all applied on $\Omega, \mathbf{u}(\mathbf{x})$ is the displacement field, at a position of $\mathbf{x}$ in the domain $\Omega$. The position in space is defined by $\mathbf{x}=\left[x_{1} ; x_{2} ; x_{3}\right]$ and $\omega$ is the angular frequency. Herein, material isotropy are assumed, and a time dependence of the form $e^{i \omega t}$ is also assumed. Generally throughout this paper, subscripts $\Sigma$ and $\Lambda$ denote the field in which the DOFs are represented. $\Sigma$ denotes all DOFs lying on the boundary denoted $\Sigma$ in Fig. 1 and $\Lambda$ denotes all DOFs not on $\Sigma . \Lambda=\Omega \cup \Gamma$ is defined as where $\Gamma=\partial \Omega \backslash \Sigma$. In other words, $\Gamma$ is the boundary which together with the boundary $\Sigma$ encloses the domain $\Omega$ and $\Lambda$ is the union of boundary $\Gamma$ and the domain $\Omega$.

\subsection{Decomposition of $\mathbf{u}(\mathbf{x})$}

The continuum mechanical displacement field, $\mathbf{u}(\mathbf{x})=\left\{u_{1}(\mathbf{x}) ; u_{2}(\mathbf{x}) ; u_{3}(\mathbf{x})\right\}$, may be written in a FE discretized representation as

$$
\mathbf{u}(\mathbf{x}) \approx \sum_{k=1}^{N}\left\{U_{3(k-1)+1}, U_{3(k-1)+2}, U_{3 k}\right\} \phi_{k}(\mathbf{x}),
$$

$N$ is the number of (generalised) nodes, $\phi_{k}(\mathbf{x})$ is the scalar global FE basis functions. Where boundary conditions on $\Gamma$ have been included in terms of constraints and loads, the constrained discretized system equations may be written in the form

$$
\left(\mathbf{K}-\omega^{2} \mathbf{M}\right) \mathbf{U}=\mathbf{F}
$$

where $\mathbf{K}$ and $\mathbf{M}$ are the stiffness and mass matrices respectively, and $\mathbf{U}$ and $\mathbf{F}$ are the displacement and force vectors respectively. Let the vector of the displacement DOFs $\mathbf{U}$ be partitioned into $\mathbf{U}_{\Sigma}$ consisting of all DOFs corresponding to global basis functions which are non-zero on $\Sigma$ and $\mathbf{U}_{\Lambda}$ consisting of the remaining DOFs corresponding to global basis functions which are zero on $\Sigma$. Let also the system matrix be partitioned correspondingly. Now, Eq. (4) may be written as

$$
\left[\left(\begin{array}{ll}
\mathbf{K}_{\Sigma \Sigma} & \mathbf{K}_{\Sigma \Lambda} \\
\mathbf{K}_{\Sigma \Lambda}^{\mathrm{T}} & \mathbf{K}_{\Lambda \Lambda}
\end{array}\right)-\omega^{2}\left(\begin{array}{ll}
\mathbf{M}_{\Sigma \Sigma} & \mathbf{M}_{\Sigma \Lambda} \\
\mathbf{M}_{\Sigma \Lambda}^{\mathrm{T}} & \mathbf{M}_{\Lambda \Lambda}
\end{array}\right)\right]\left(\begin{array}{c}
\mathbf{U}_{\Sigma} \\
\mathbf{U}_{\Lambda}
\end{array}\right)=\left(\begin{array}{l}
\mathbf{F}_{\Sigma} \\
\mathbf{F}_{\Lambda}
\end{array}\right) .
$$




\subsection{Change of basis}

This subsection explains how to generate the (nodal) displacement vector representation of the basis functions used in the change basis CMS approach. The Classical C\&B substructuring method uses one set of basis functions to span the local DOF of each substructure, and another set of functions for the coupling of the structures. The two sets of functions will be referred to here as local modes and coupling modes.

\subsubsection{Local modes}

The local modes are generated by a constrained eigenvalue problem. On the coupling interface $\Sigma$ a homogeneous (zero) Dirichlet condition is always imposed as $\mathbf{U}_{\Sigma}=\mathbf{0}$ together with zero force conditions on both $\Sigma$ and $\Gamma$. Hence, the eigenvalue problem may be written as

$$
\left(\mathbf{K}_{\Lambda \Lambda}-\omega^{2} \mathbf{M}_{\Lambda \Lambda}\right) \mathbf{U}_{\Lambda}=\mathbf{0} .
$$

Where any homogeneous Dirichlet condition imposed on $\Gamma$ in the original boundary value problem (BVP), is also satisfied in the eigenvalue problem.

The solution of Eq. (5) gives the eigenvalues $\omega_{n}^{2}$ and eigenvectors $\phi_{n}$. The total displacement vectors of the constrained eigenvalue problem, including the constrained coupling interface DOFs as zeroes, is written as in Eq. (6) where each column corresponds to a displacement eigenvector

$$
\boldsymbol{\Phi}=\left(\begin{array}{c}
\mathbf{0} \\
\phi_{\Lambda,(1)} \cdots \phi_{\Lambda,(n)}
\end{array}\right)=\left(\begin{array}{c}
\mathbf{0} \\
\boldsymbol{\Phi}_{\Lambda}
\end{array}\right) .
$$

This is the first set of basis functions used in the projection from local DOFs to generalised DOFs.

\subsubsection{Coupling modes}

To allow for kinematic coupling to an adjacent substructure, basis functions which are non-zero on $\Sigma$ are needed. These are constructed by static solutions of $M^{\text {coup }}$, different linearly independent BVPs having different non-zero prescribed displacement conditions on $\Sigma$. These boundary constraints are imposed via the FE displacement vector $\mathbf{U}_{\Sigma}=\psi_{\Sigma,(m)}$ where $\mathbf{F}_{\Sigma}=\mathbf{0}$ and $\mathbf{F}_{\Lambda}=\mathbf{0}$. Denoting the corresponding solution of $\mathbf{U}_{\Lambda}$ of the remaining displacement DOFs by $\mathbf{U}_{\Lambda}=\psi_{\Lambda,(m)}$, the $m$ th solution is obtained by solving the static problem

$$
\left(\begin{array}{ll}
\mathbf{K}_{\Sigma \Sigma} & \mathbf{K}_{\Sigma \Lambda} \\
\mathbf{K}_{\Sigma \Lambda}^{\mathrm{T}} & \mathbf{K}_{\Lambda \Lambda}
\end{array}\right)\left(\begin{array}{l}
\psi_{\Sigma,(m)} \\
\psi_{\Lambda,(m)}
\end{array}\right)=\left(\begin{array}{l}
\mathbf{0} \\
\mathbf{0}
\end{array}\right)
$$

hence,

$$
\psi_{\Lambda,(m)}=-\mathbf{K}_{\Lambda \Lambda}^{-1} \mathbf{K}_{\Sigma \Lambda}^{\mathrm{T}} \psi_{\Sigma,(m)} .
$$

The $M^{\text {coup }}$ solutions of Eq. (8) may be organised as Eq. (9) where each column corresponds to the displacement vector (or "mode") for a given imposed displacement vector $\psi_{\Sigma,(m)}$.

$$
\boldsymbol{\Psi}=\left(\begin{array}{l}
\boldsymbol{\Psi}_{\Sigma} \\
\boldsymbol{\Psi}_{\Lambda}
\end{array}\right)=\left(\begin{array}{lll}
\psi_{\Sigma,(1)} & \cdots & \psi_{\Sigma,(m)} \\
\psi_{\Lambda,(1)} & \cdots & \psi_{\Lambda,(m)}
\end{array}\right)
$$

This is the second set of basic functions used in the projection from local DOFs to generalised DOFs. 


\subsubsection{Modal projection}

If the two projection basis functions of the local and the coupling modes are assembled, the total projection basic $\Theta$ reads

$$
\boldsymbol{\Theta}=[\boldsymbol{\Psi}, \boldsymbol{\Phi}]=\left(\begin{array}{cc}
\boldsymbol{\Psi}_{\Sigma} & \mathbf{0} \\
\boldsymbol{\Psi}_{\Lambda} & \boldsymbol{\Phi}_{\Lambda}
\end{array}\right)
$$

The projection of each component of Eq. (3) may be written as

$$
\mathbf{S}=\Theta^{\mathrm{T}} \mathbf{K} \Theta, \quad \mathbf{W}=\boldsymbol{\Theta}^{\mathrm{T}} \mathbf{M} \Theta
$$

and

$$
\mathbf{G}=\Theta^{\mathrm{T}} \mathbf{F}, \quad \mathbf{U}_{\text {modal }}=\Theta \mathbf{Q}
$$

and, as a result, the original nodal forces have accordingly to be transformed to modal forces as

$$
\left(\begin{array}{c}
\mathbf{G}_{\Sigma} \\
\mathbf{G}_{\Lambda}
\end{array}\right)=\left(\begin{array}{c}
\boldsymbol{\Psi}_{\Sigma}^{\mathrm{T}} \mathbf{F}_{\Sigma}+\boldsymbol{\Psi}_{\Lambda}^{\mathrm{T}} \mathbf{F}_{\Lambda} \\
\mathbf{\Phi}_{\Lambda}^{\mathrm{T}} \mathbf{F}_{\Lambda}
\end{array}\right)
$$

with the following transformed system of generalised coordinates:

$$
\left[\left(\begin{array}{cc}
\mathbf{S}_{\Sigma \Sigma} & \mathbf{0} \\
\mathbf{0} & \mathbf{S}_{\Lambda \Lambda}
\end{array}\right)-\omega^{2}\left(\begin{array}{ll}
\mathbf{W}_{\Sigma \Sigma} & \mathbf{W}_{\Sigma \Lambda} \\
\mathbf{W}_{\Sigma \Lambda}^{\mathrm{T}} & \mathbf{W}_{\Lambda \Lambda}
\end{array}\right)\right]\left(\begin{array}{l}
\mathbf{Q}_{\Sigma} \\
\mathbf{Q}_{\Lambda}
\end{array}\right)=\left(\begin{array}{l}
\mathbf{G}_{\Sigma} \\
\mathbf{G}_{\Lambda}
\end{array}\right)
$$

If the eigenvectors $\phi_{\Lambda}$ in the projection basis are mass normalised then $\mathbf{W}_{\Lambda \Lambda}$ is a diagonal matrix with only ones in the diagonal, $\mathbf{S}_{\Lambda \Lambda}$ is a diagonal matrix with the eigenvalues $\omega_{n}^{2}$ in the diagonal. The mass and the stiffness submatrices of the interface DOFs, $\mathbf{W}_{\Sigma \Sigma}, \mathbf{S}_{\Sigma \Sigma}$ are full matrices, and the coupling (interface or inner DOFs) mass submatrix $\left(\mathbf{W}_{\Sigma \Lambda}\right)$ is also full. Hence, the global mass matrix $\mathbf{W}$ is also full. However, the fast solution properties of the diagonal matrix $\mathbf{W}_{\Lambda \Lambda}$ can still be utilised by condensing the solution to interface DOFs.

\subsubsection{Classical Craig-Bampton}

In Eq. (14) the general form of the classical C\&B method is formulated. For the special case when $\Psi_{\Sigma}$ is chosen as the identity matrix I then Eq. (14) corresponds exactly to the classical C\&B method. That formulation is based on the calculation of the inner response (on $\Lambda$ ) for the case of successively displacing each DOF (on $\Sigma$ ) by a unity while keeping all other DOFs on the interface fixed. This procedure of repeating the calculation of Eq. (8) as many times at there are DOFs on $\Sigma$, is equivalent to replacing $\boldsymbol{\Psi}_{\Sigma}$ with the unit matrix. Hence, no reduction of interface $(\Sigma)$ DOFs is made, since multiplying by a square unit matrix will not change the dimension of the resulting matrix. Another common notation is that modal forces are not allowed and hence, all body forces are put in the unreduced boundaries as in

$$
\left[\left(\begin{array}{cc}
\mathbf{S}_{\Sigma \Sigma} & \mathbf{0} \\
\mathbf{0} & \mathbf{S}_{\Lambda \Lambda}
\end{array}\right)-\omega^{2}\left(\begin{array}{ll}
\mathbf{W}_{\Sigma \Sigma} & \mathbf{W}_{\Sigma \Lambda} \\
\mathbf{W}_{\Sigma \Lambda}^{\mathrm{T}} & \mathbf{W}_{\Lambda \Lambda}
\end{array}\right)\right]\left(\begin{array}{l}
\mathbf{U}_{\Sigma} \\
\mathbf{Q}_{\Lambda}
\end{array}\right)=\left(\begin{array}{c}
\mathbf{F}_{\Sigma} \\
\mathbf{0}
\end{array}\right)
$$

\subsection{Coupling of structures}

In this paper only the formulation of the coupling between two substructures is; shown the general problem of more substructures follows naturally. In Fig. 2 the coupled problem of two substructures is presented using the same formalism as in Fig. $1 \Sigma=\partial \Omega_{r} \backslash \Gamma_{r}$ or $\Sigma=\partial \Omega_{1} \cap \partial \Omega_{2}$, and $\Lambda_{r}=\Omega_{r} \cup \Gamma_{r}$.

The directly coupled equation in FE form can be written as Eq. (16) using the same organisation of the matrices as in Eq. (4). Conforming meshes of the substructures on $\Sigma$ is here assumed

$$
\left[\left(\begin{array}{rrr}
\mathbf{K}_{\Sigma \Sigma}^{(1)}+\mathbf{K}_{\Sigma \Sigma}^{(2)} & \mathbf{K}_{\Sigma \Lambda}^{(1)} & \mathbf{K}_{\Sigma \Lambda}^{(2)} \\
& \mathbf{K}_{\Lambda \Lambda}^{(1)} & \mathbf{0} \\
\operatorname{sym} . & \mathbf{K}_{\Lambda \Lambda}^{(2)} &
\end{array}\right)-\omega^{2}\left(\begin{array}{ccc}
\mathbf{M}_{\Sigma \Sigma}^{(1)}+\mathbf{M}_{\Sigma \Sigma}^{(2)} & \mathbf{M}_{\Sigma \Lambda}^{(1)} & \mathbf{M}_{\Sigma \Lambda}^{(2)} \\
& \mathbf{M}_{\Lambda \Lambda}^{(1)} & \mathbf{0} \\
\operatorname{sym} . & \mathbf{M}_{\Lambda \Lambda}^{(2)} &
\end{array}\right)\right]\left(\begin{array}{c}
\mathbf{U}_{\Sigma} \\
\mathbf{U}_{\Lambda}^{(1)} \\
\mathbf{U}_{\Lambda}^{(2)}
\end{array}\right)=\left(\begin{array}{c}
\mathbf{F}_{\Sigma} \\
\mathbf{F}_{\Lambda}^{(1)} \\
\mathbf{F}_{\Lambda}^{(2)}
\end{array}\right)
$$


The subscript in parentheses indicates which substructure the DOFs belong to. By a change of basis Eq. (17) the FE displacement vector can be rewritten as

$$
\left(\begin{array}{c}
\mathbf{U}_{\Sigma} \\
\mathbf{U}_{\Lambda}^{(1)} \\
\mathbf{U}_{\Lambda}^{(2)}
\end{array}\right)=\left(\begin{array}{ccc}
\boldsymbol{\Psi}_{\Sigma} & \mathbf{0} & \mathbf{0} \\
\mathbf{\Psi}_{\Lambda}^{(1)} & \boldsymbol{\Phi}_{\Lambda}^{(1)} & \mathbf{0} \\
\mathbf{\Psi}_{\Lambda}^{(2)} & \mathbf{0} & \boldsymbol{\Phi}_{\Lambda}^{(2)}
\end{array}\right)\left(\begin{array}{c}
\mathbf{Q}_{\Sigma} \\
\mathbf{Q}_{\Lambda}^{(1)} \\
\mathbf{Q}_{\Lambda}^{(2)}
\end{array}\right)
$$

Note that $\boldsymbol{\Psi}_{\Sigma}$ has no substructure superscript. This is because only one $\boldsymbol{\Psi}_{\Sigma}$ matrix containing the pre-prescribed displacements is needed for each coupling interface $\Sigma$ (if the mesh conforms at the interface). Hence, the same $\boldsymbol{\Psi}_{\Sigma}$ is used in the computation of all $\Psi_{\Lambda}^{(r)}$ that share the interface $\Sigma$. The transformed system equations may be written as

$$
\left[\left(\begin{array}{ccc}
\mathbf{S}_{\Sigma \Sigma} & \mathbf{0} & \mathbf{0} \\
& \mathbf{S}_{\Lambda \Lambda}^{(1)} & \mathbf{0} \\
\text { sym. } & \mathbf{S}_{\Lambda \Lambda}^{(2)} &
\end{array}\right)-\omega^{2}\left(\begin{array}{ccc}
\mathbf{W}_{\Sigma \Sigma} & \mathbf{W}_{\Sigma \Lambda}^{(1)} & \mathbf{W}_{\Sigma \Lambda}^{(2)} \\
& \mathbf{W}_{\Lambda \Lambda}^{(1)} & \mathbf{0} \\
\text { sym. } & \mathbf{W}_{\Lambda \Lambda}^{(2)} &
\end{array}\right)\right]\left(\begin{array}{c}
\mathbf{Q}_{\Sigma} \\
\mathbf{Q}_{\Lambda}^{(1)} \\
\mathbf{Q}_{\Lambda}^{(2)}
\end{array}\right)=\left(\begin{array}{c}
\mathbf{G}_{\Sigma} \\
\mathbf{G}_{\Lambda}^{(1)} \\
\mathbf{G}_{\Lambda}^{(2)}
\end{array}\right)
$$

If the eigenvectors $\boldsymbol{\Phi}_{\Lambda}^{(r)}$ is mass normalised then the diagonal matrices $\mathbf{S}_{\Lambda \Lambda}^{(r)}$ and $\mathbf{W}_{\Lambda \Lambda}^{(r)}$ may be written as

$$
\mathbf{S}_{\Lambda \Lambda}^{(r)}=\left(\begin{array}{cccc}
\omega_{1}^{2} & 0 & \cdots & 0 \\
0 & \omega_{2}^{2} & \cdots & 0 \\
\vdots & \vdots & \ddots & \vdots \\
0 & 0 & \cdots & \omega_{n}^{2}
\end{array}\right) \quad \mathbf{W}_{\Lambda \Lambda}^{(r)}=\left(\begin{array}{cccc}
1 & 0 & \cdots & 0 \\
0 & 1 & \cdots & 0 \\
\vdots & \vdots & \ddots & \vdots \\
0 & 0 & \cdots & 1
\end{array}\right)
$$

where $\omega_{n}^{2}$ indicates the corresponding eigenvalues from the local modes.

The submatrix of the stiffness matrix $\mathbf{S}_{\Sigma \Sigma}$ may then be expressed as

$$
\mathbf{S}_{\Sigma \Sigma}=\sum_{r=1}^{2} \boldsymbol{\Psi}_{\Sigma}^{\mathrm{T}} \mathbf{K}_{\Sigma \Sigma}^{(r)} \boldsymbol{\Psi}_{\Sigma}+\sum_{r=1}^{2} \boldsymbol{\Psi}_{\Lambda}^{(r) \mathrm{T}} \mathbf{K}_{\Lambda \Sigma}^{(r)} \boldsymbol{\Psi}_{\Sigma}
$$

and the submatrix of the mass matrix $\mathbf{W}_{\Sigma \Sigma}$ may be expressed as

$$
\mathbf{W}_{\Sigma \Sigma}=\sum_{r=1}^{2} \boldsymbol{\Psi}_{\Sigma}^{\mathrm{T}} \mathbf{M}_{\Sigma \Sigma}^{(r)} \boldsymbol{\Psi}_{\Sigma}+\sum_{r=1}^{2} \boldsymbol{\Psi}_{\Sigma}^{\mathrm{T}} \mathbf{M}_{\Sigma \Lambda}^{(r)} \boldsymbol{\Psi}_{\Lambda}^{(r)}+\sum_{r=1}^{2} \boldsymbol{\Psi}_{\Lambda}^{(r) \mathrm{T}} \mathbf{M}_{\Lambda \Lambda}^{(r)} \mathbf{\Psi}_{\Lambda}^{(r)}+\sum_{r=1}^{2} \boldsymbol{\Psi}_{\Lambda}^{(r) \mathrm{T}} \mathbf{M}_{\Lambda \Sigma}^{(r)} \boldsymbol{\Psi}_{\Sigma}
$$

Finally, the submatrix for the coupling in the mass matrix $\mathbf{W}_{\Sigma \Lambda}$ can then be expressed as

$$
\mathbf{W}_{\Sigma \Lambda}^{(r)}=\boldsymbol{\Psi}_{\Lambda}^{(r) \mathrm{T}} \mathbf{M}_{\Lambda \Lambda}^{(r)} \boldsymbol{\Phi}_{\Lambda}^{(r)}+\boldsymbol{\Psi}_{\Sigma}^{\mathrm{T}} \mathbf{M}_{\Sigma \Lambda}^{(r)} \boldsymbol{\Phi}_{\Lambda}^{(r)}=\mathbf{W}_{\Lambda \Sigma}^{(t) \mathrm{T}} .
$$

The computation of a large system consisting of numerous substructures may well be condensed to only computing the displacement of the interface DOFs.

\subsubsection{Six DOFs for rigid interfaces}

So far only a general form of the classical C\&B CMS has been presented. In the theory presented herein the possibility of using any function that can be described on $\Sigma$ has been shown, in order to calculate the static coupling modes $\psi$. As stated before, in the classical formulation of the C\&B CMS an unreduced interface formulation is used.The $\mathrm{C} \& \mathrm{~B}$ formulation is based on the computation of the inner response (on $\Lambda$ ) by successively displacing each DOF by a unity while keeping all other DOFs on the interface fixed. In this paper a different set of displacement conditions is constructed to generate a different set of static coupling modes with Eq. (8).

Herein, a undeformed coupling interface (UCI) approach is introduced. The way in which an interface between two connected bodies deforms could be argued to be governed (among other parameters) by the relative stiffness of the bodies. If there is a large relative difference in the stiffness, then the coupling interface may translate and rotate, 
but keeping its original shape almost undeformed. The stiffer body interface behaves almost as a free boundary and the softer body interfaces behave similarly to a spatially prescribed translation and rotation, that is, identical to the stiffer body translation and rotation.

These assumptions of undeformed coupling interfaces allow for a restriction of coupling modes to represent six different rigid motions of the interface, e.g. three translations and three rotations around a common rotation point. In all other aspects, these coupling modes correspond to the classical C\&B coupling modes. The main advantage of this approach is that the number of coupling modes are reduced from the number of FE DOFs associated to the coupling boundary $\Sigma$, to six. Another important feature is that mesh compatibility is not required: only the translation directions, rotation axes and rotation point have to be compatible. Formally, not even the geometry has to be compatible.

The three orthogonal constant translations in space could be written as

$$
\psi_{\Sigma}^{(q)}=\frac{\Delta \mathbf{x}_{\Sigma}^{(q)}}{\left\|\Delta \mathbf{x}_{\Sigma}^{(q)}\right\|_{2}}, \quad q=[1,2,3]
$$

for,

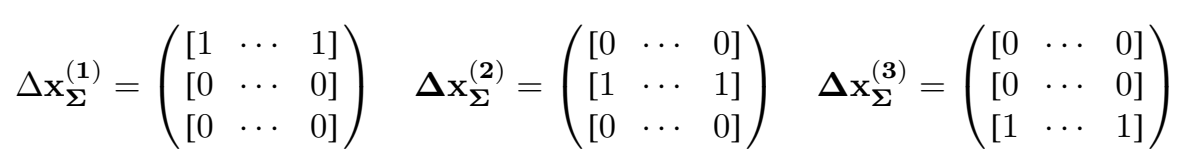

where $\Delta \mathbf{x}_{\Sigma}$ is the displacement from the original position $\mathbf{x}_{\Sigma}$, which is the matrix containing the nodal coordinates on $\Sigma$, and each row corresponds to a spatial direction. The matrix only giving the displacement from the original positions may then be written as

$$
\psi_{\Sigma}^{(q+3)}=\frac{\mathbf{R}_{x_{q}}^{*} \mathbf{x}_{\Sigma}}{\left\|\mathbf{R}_{x_{q}}^{*} \mathbf{x}_{\Sigma}\right\|_{2}}, \quad q=[1,2,3]
$$

where, $\mathbf{R}_{x_{q}}^{*}=\mathbf{R}_{x_{q}}-\mathbf{I}$ where $\mathbf{I}$ is the three by three unit matrix, and $\mathbf{R}_{x_{q}}$ corresponds to the matrix which projects a rotation about a fixed axis in space, for a given angle $\alpha$ which may be written as

$$
\mathbf{R}_{x_{1}}^{*}=\left(\begin{array}{ccc}
0 & 0 & 0 \\
0 & 0 & -\alpha \\
0 & \alpha & 0
\end{array}\right) \quad \mathbf{R}_{x_{2}}^{*}=\left(\begin{array}{ccc}
0 & 0 & \alpha \\
0 & 0 & 0 \\
-\alpha & 0 & 0
\end{array}\right) \quad \mathbf{R}_{x_{3}}^{*}=\left(\begin{array}{ccc}
0 & -\alpha & 0 \\
\alpha & 0 & 0 \\
0 & 0 & 0
\end{array}\right)
$$

valid for small angles of $\alpha$.

\section{Results and discussion}

In order to evaluate the usefulness of the proposed approach a test structure is implemented. The question is whether the approach with the UCI may be used for vibro-acoustic modelling of built up substructures of fundamentally different stiffnesses.

\subsection{Test structure}

The test structure is shown in Fig. 3. The properties of this test case are chosen to resemble the vibro-acoustic problem of a triangular linking arm in a vehicle suspension system, the linking arm often being connected to the stiff vehicle body in three positions via rubber bushings. The test structure consists of a cross (Substructure 3 in Fig. 3) to resemble the linking arm. The rubber bushings are included as blocks (Substructures 2, 4 and 6 in Fig. 3) and three connecting parts are included (Substructures 1, 5 and 7 in Fig. 3) to mimic the stiffness of the vehicle body. The material properties are listed in Table 1 . The geometrical properties of the three stiff dynamic connectors 
Table 1

Material properties used in the test structure. The damping included is proportional damping

\begin{tabular}{|c|c|c|c|c|}
\hline Sub Nr. & Young's modulus & Density & Poisson's ratio & Damping \\
\hline 1 & $100[\mathrm{GPa}]$ & $7850\left[\mathrm{~kg} / \mathrm{m}^{3}\right]$ & $0.33[-]$ & $0.01[-]$ \\
\hline 2 & $100[\mathrm{kPa}]^{\dagger}$ & $1100\left[\mathrm{~kg} / \mathrm{m}^{3}\right]$ & $0.45[-]$ & $0.10[-]$ \\
\hline 3 & 100 [GPa] & $7850\left[\mathrm{~kg} / \mathrm{m}^{3}\right]$ & $0.33[-]$ & $0.01[-]$ \\
\hline 4 & $100[\mathrm{kPa}]^{\dagger}$ & $1100\left[\mathrm{~kg} / \mathrm{m}^{3}\right]$ & $0.45[-]$ & $0.10[-]$ \\
\hline 5 & $100[\mathrm{GPa}]$ & $7850\left[\mathrm{~kg} / \mathrm{m}^{3}\right]$ & $0.33[-]$ & $0.01[-]$ \\
\hline 6 & $100[\mathrm{kPa}]^{\dagger}$ & $1100\left[\mathrm{~kg} / \mathrm{m}^{3}\right]$ & $0.45[-]$ & $0.10[-]$ \\
\hline 7 & $100[\mathrm{GPa}]$ & $7850\left[\mathrm{~kg} / \mathrm{m}^{3}\right]$ & $0.33[-]$ & $0.01[-]$ \\
\hline
\end{tabular}

$\dagger$ In Figs 7 and 8 a varying Young's module is used for the three "soft" connectors.

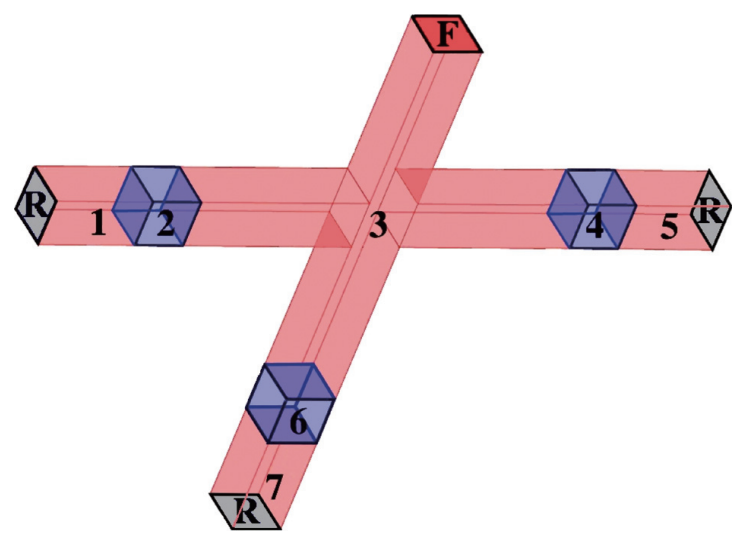

Fig. 3. The test structure used in this paper consisting of seven substructures. Boundary conditions are indicated by R (Rigid or Fixed) or F (unit surface force condition in all the spatial directions).

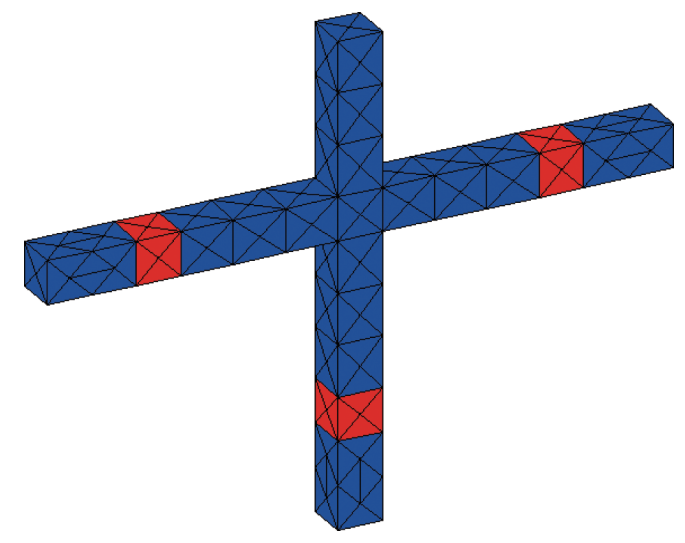

Fig. 4. The mesh used on the test structure in this paper. The soft connectors are indicated with red.

representing the vehicle body have dimensions $0.1 \times 0.05 \times 0.05 \mathrm{~m}$. All three have a fixed (rigid) boundary condition on the boundary opposite to the connection interface. The rigid boundary is indicated by an R in Fig. 3. The soft connectors are cubes of dimension $0.05 \times 0.05 \times 0.05 \mathrm{~m}$. The cross is symmetric with the outer dimension of the "arms" $0.4 \times 0.4 \mathrm{~m}$. Hence, each of the four arms has dimensions $0.175 \times 0.05 \times 0.05 \mathrm{~m}$, three of the arms are connected to the three soft connectors and the fourth has a unit surface force boundary condition (a unit surface force in each spatial direction) as indicated by the surface with an F in Fig. 3.

The mesh used in the evaluation is shown in Fig. 4; second-order Lagrange polynomials are used as the basis functions of the original problem. The true solution of the problem is not relevant for this study; only a comparative solution able to evaluate the possibility of using a reduction method on the local FE problems is desired. In other words, the test case is defined as the numerical representation of the geometry imposed by the FE-discretisation, and not the physical problem. Hence the solution of the original problem needs not be converged. However, using the same mesh but going to a third order polynomial basis of the original global problem, the relative difference off resonance is in the order of $1 \%$. The error are higher at resonance due to the inherent problem of ill-conditioned matrices at resonance. The original numerical problem consists 339 DOFs of each substructure for Substructures 1, 5 and 7, and for Substructures 2, 4 and 6 it consists of 189 DOFs and Substructure 3 consists of 1749 DOFs. Due to boundary conditions and coupling interfaces this gives the possibility of 291 normal local modes for Substructure 1 , 5 and 7; 141 for subs 2, 5, and 6 and 1677 modes for sub 3.

\subsection{Vibro-acoustic response}

Figure 5 shows a representation of the vibro-acoustic behaviour of the above described problem. The solid line shows the total displacement of Substructure 3 in an integral sense (RMS value of L2 norm) for the direct FE solution. The dashed line illustrates the mass law frequency slope; the starting value of the line is adjusted to show the clear mass law dependence of the solid line in the range of approximately $10-600 \mathrm{~Hz}$. It may be seen in 


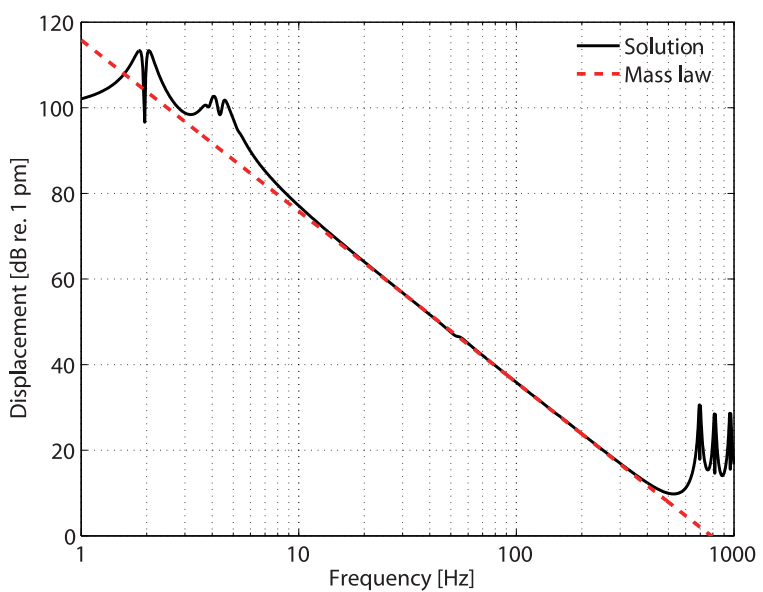

Fig. 5. The vibro-acoustic response, integral of the total displacement of Substructure 3 (solid line), together with a visualisation line showing a mass law frequency dependence (dashed line).

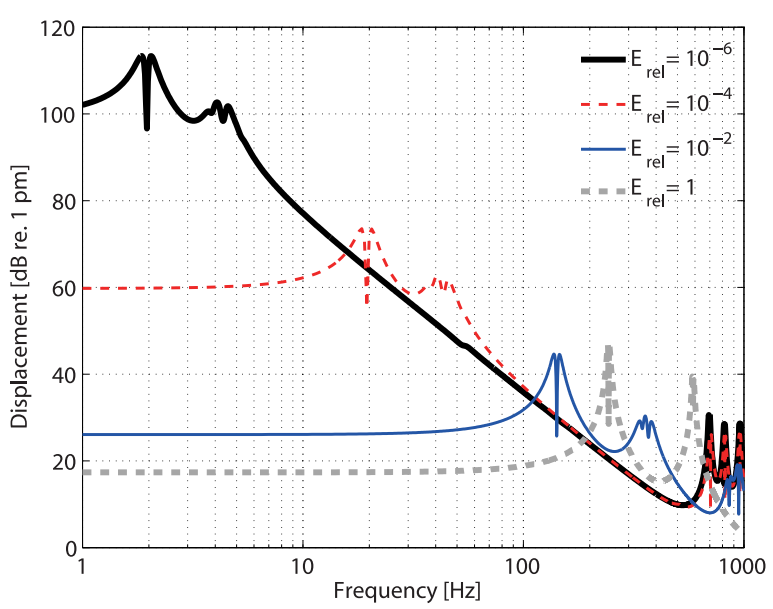

Fig. 7. The vibro-acoustic response, integral of the total displacement of Substructure 3 for four different Young's module of the soft connectors, presented as the relative Young's modulus.

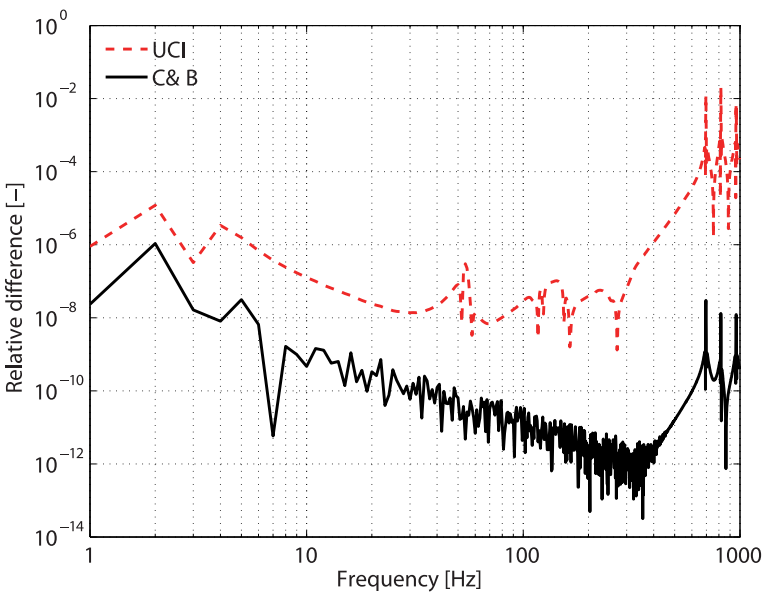

Fig. 6. Relative difference between the direct solution and the C\&B method (solid line) and the UCI approach (dashed line), both cases using all inner modes.

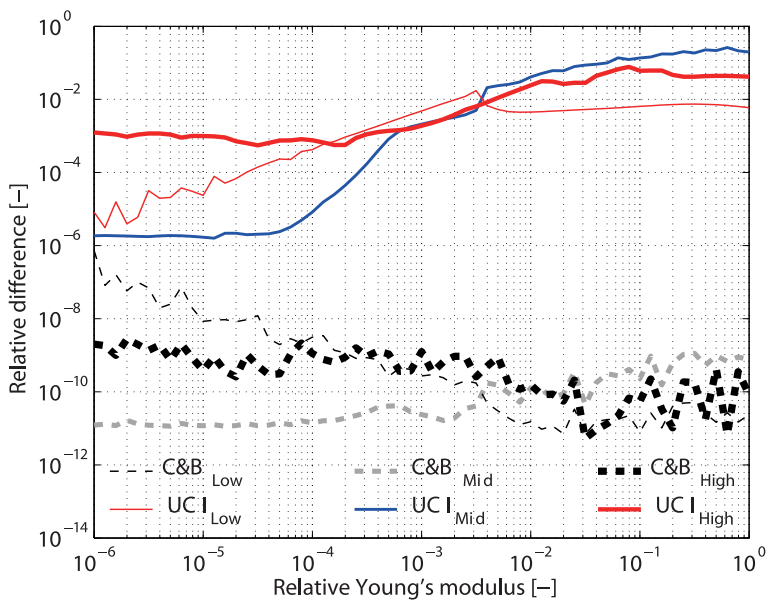

Fig. 8. The total relative difference between the modal solutions and the direct solution for the three frequency bands, using all inner modes. Function of the stiffness of the "soft" contactors (function of relative stiffness). C\&B method (dashed lines) UCI approach (solid lines).

Fig. 5 that there are three frequency regions of different behaviour of the solution. In the low frequency range is the rigid body resonance behaviour of Substructure 3. At mid frequencies the behaviour is mostly governed by the mass law, and at high frequencies there are three elastic modes of the cross that governs the behaviour. Figure 6 shows the relative difference between the direct FE solution and the C\&B method (solid line) and the UCI approach (dashed line). Both the solutions for C\&B and UCI are computed for the case when no reduction of inner modes is made. Hence, no reduction at all is made for $\mathrm{C} \& \mathrm{~B}$ while an interface reduction is made for the UCI approach. What can firstly be noted in Fig. 6 is the effect of round-off errors, (see solid line C\&B) which may be enhanced due to the large difference in Young's modulus $\left(\mathrm{E}_{\text {soft }} / \mathrm{E}_{\text {stiff }}=\mathrm{E}_{\text {rel }}=10^{-6}\right)$. Furthermore, a limit of the relative difference for the $\mathrm{C} \& \mathrm{~B}$ method of around $10^{-12}$ (off resonance) is observed, (smaller deviations are not observed). At resonance, deviations are larger because of the ill-conditioned system matrix. It should be noted that even though the relative difference is larger for the UCI approach, from an engineering perspective, the results are still good, and the difference is still smaller than the error imposed by the original FE discretisation.

From Figs 5 and 6 three different frequency regions can be recognised, as discussed previously. These are defined 


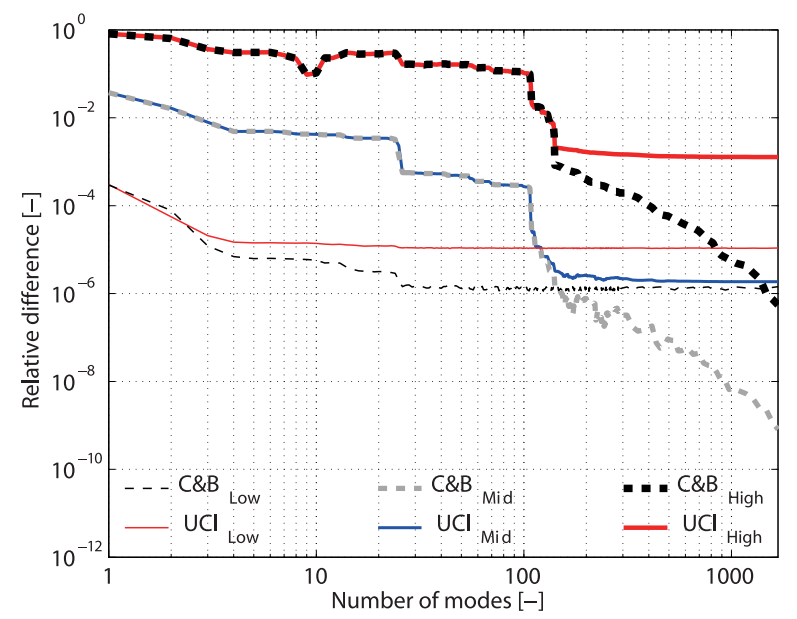

Fig. 9. The total relative difference between the modal solutions and the direct solution for the three frequency bands, plotted as a function of the number of included modes inner in each substructure. $C \& B$ method (dashed lines) UCI approach (solid lines).

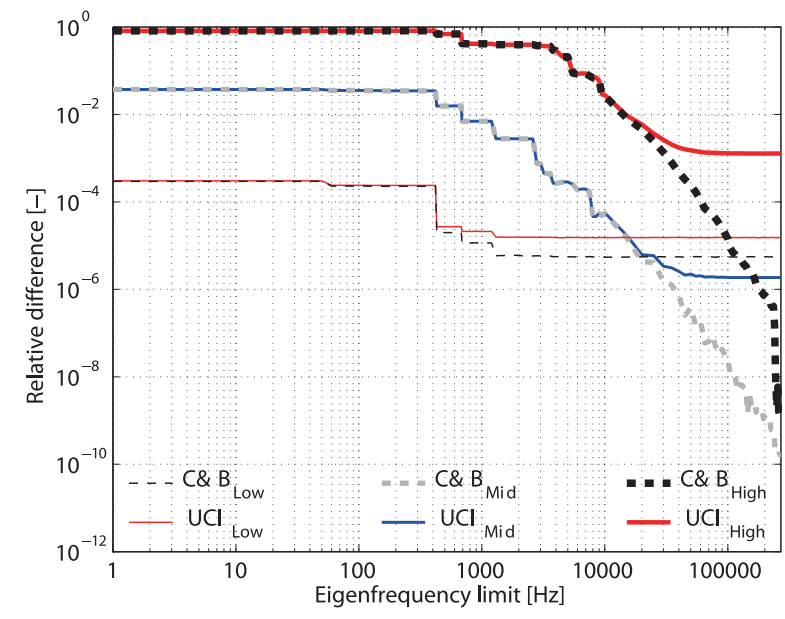

Fig. 10. The total relative difference between the modal solutions and the direct solution for the three frequency bands, plotted as a function of the eigenfrequency limit of included inner modes. C\&B method (dashed lines) UCI approach (solid lines).

as low $(0-100 \mathrm{~Hz})$, mid $(100-600 \mathrm{~Hz})$ and high $(600-1000 \mathrm{~Hz})$, mainly for evaluation purposes. In Fig. 7 the solution defined as above is plotted for different Young's modulus of the "soft" connectors. When the relative Young's modulus moves closer to unity, then the first rigid body resonance moves up in frequency (see Fig. 7).

\subsection{Evaluation of the approach}

\subsubsection{Relative Young's modulus}

In Fig. 8 the overall relative difference in each previously defined frequency range (low, mid and high) is plotted for changing stiffness of the "soft" connectors. In other words all material properties are kept constant except the Young's modulus of the Substructures 2, 4 and 6. What can be seen in Fig. 8 is that, in all cases, C\&B and UCI show results that may be considered as accurate from an engineering point of view, with deviation less the $1 \%$, for a relative Young's modulus $\mathrm{E}_{\text {rel }}$ of less the of around $10^{-3}$. When evaluating the result in Fig. 8 it should be noted that resonances are shifted between different frequency bands; thus, the ill-conditioned behaviour of these resonances is also shifted between the bands (low, mid and high see Fig. 7). The solution of the C\&B method is as expected good for all relative Young's modulus, even though at high and low frequencies the results are less accurate for a very low relative Young's modulus. This is probably due to the resonant behaviour and the fact that large jumps in Young's modulus may impose an ill-conditioned system matrix. As expected, higher frequencies are hardest to predict with the UCI approach, probably because at high frequency the size of the interface become large in comparison to the wave length. In this test case, a fairly large interface was used, whereas in a vehicle, the interfaces between structures and bushings are seldom large. The test case evaluated herein should only be viewed as a test of the possibility of modelling problems remotely similar to the built up structure of softer and stiffer parts, such as vehicle suspensions. The possibility of using the UCI approach for more general cases has to be further evaluated. So far, only the results including all modes for the $\mathrm{C} \& \mathrm{~B}$ and UCI approaches have been shown, which highlights the consequences of the UCI approach compared to the C\&B method.

\subsubsection{Inner reduction}

In Fig. 9 the solution is calculated with the original material properties $\left(\mathrm{E}_{r e l}=10^{-6}\right)$, and the relative total difference for the different frequency bands is plotted as a function of the number of included normal modes of each substructure. In other words the results are generated by calculating the response when one normal mode is included in each substructure and then using two modes in each and so on until all modes are filled up in all substructures (the modes are organised by ascending eigenfrequency). From Fig. 9 it should be noted that, when using a low number of modes (less then 100), the low frequency representation is better than mid and mid is better than high, as 
expected from a wavelength perspective. Furthermore, it is clear that for a low number of modes the results for $\mathrm{C} \& \mathrm{~B}$ and UCI are very similar up to around $100+$ modes, except for the low frequency range where the C\&B result is somewhat better for very few modes. It seems that the UCI results have a cut-on limit, where any refined description of the inner problem no longer enhances the results; the limiting factor is most likely due to the error induced by the interface reduction. However, in this specific case the limit of the relative difference due to truncation of the interface DOFs seems to be below an engineering error $(\sim 1 \%)$. It should also be noted that the limit for the relative difference of the UCI is not only governed by the frequency but also by the resonant behaviour of the frequency at hand. Moreover, the low frequency C\&B also seems to have some sort of cut-on limit, whereas the mid and high have a very steep relative difference curve in the end of the curve, when all modes are filled up in the model. This behaviour might be due to the fact that the (ill-conditioned) rigid body resonances at low frequency do not become better represented by adding higher frequency modes. In addition, it is interesting to see that the result for the mid and high frequency has a region where the result improves faster, and that this region coincides well with when the "soft" connectors have been filled up with all normal modes (141 possible modes).

Reduction methods in acoustics often use the eigenfrequency as a criterion to decide on how many modes to include. Such criteria may often be formulated such that all modes with an eigenfrequency below a specific frequency limit should be added; the frequency limit is often a factor larger than one multiplied by the highest frequency of interest. The approach of the classical C\&B (also used for the UCI) to generate local normal modes, for fixed boundary conditions of the coupling interfaces makes this frequency limit less obvious. The local modes will naturally be higher in frequency (since they are fixed) than the global modes of the coupled problem. Consequently, it is natural to suspect that a higher factor is needed for these types of methods.

In Fig. 10 the relative differences (between direct FE and C\&B, UCI) is plotted for cases in Fig. 9 but now as a function of eigenfrequency limit. In other words, for a set of different eigenfrequency limits, the result is generated when including all local normal modes below that frequency limit. It should be noted that always at least one mode is included in each substructure. It can be noted that the slopes of the high and mid frequency curves are very similar. Thus no clear tendency to a frequency limit correlated to the maximum frequency of interest can be seen. It seems that roughly the same modes influence the relative result of the mid and high frequencies similarly, where the big difference is found at the minimum of included modes (one in each substructure) where the mid frequency representation is better than the high. For a given relative difference of, for instance, $10^{-2}$, the frequency factor needed to ensure this result seems to be fundamentally different for the high- and the mid-frequency band: whereas a factor of about 10 is needed for the high-frequency band, the limit for the mid-frequency band seems to be in the order of 1 . It should be noted here that the mid-frequency band has no dynamic resonance in the response, and therefore modal representation of the problem probably needs fewer dynamic modes. It is clear that for low frequency a high limit of the eigenfrequency is not necessary i.e. a static representation is sufficient.

\subsubsection{Time gain}

As mentioned earlier there are possible benefits of the UCI, such as, that strictly neither mesh conformity nor geometrical conformity are required. However, the maybe biggest gain is the reduced computation effort needed with UCI approach. In Fig. 11 the normalised time consumption is plotted as a function of number of frequencies calculated. The time is recorded for four different sets of number of frequencies calculated, that is, 11, 101, 1001 and 10001. Seven different cases are evaluated C\&B and UCI for all, 10 and 100 modes and finally the direct FE solution. The plotted time is the time to assemble the global problem for local stiffness and mass matrices, solve it and back substitute it to the original DOFs. Furthermore, the time is normalised with the total time of the direct solution for 11 frequencies calculated. What can be seen in Fig. 11 is that for all cases the UCI approach have a faster solution time than the $\mathrm{C} \& \mathrm{~B}$ method, it is also clear that the more frequencies that are calculated the more efficient the UCI approach is compared to both C\&B and the direct solution. As expected, the fewer local inner modes used, the more efficient both C\&B and UCI is compared to the direct solution.

\subsection{Application}

To show the usefulness and the simplicity of the proposed approach, a more industrially applicable geometry was implemented. This model geometry demonstrates the simplicity of coupling separate FE models together where 


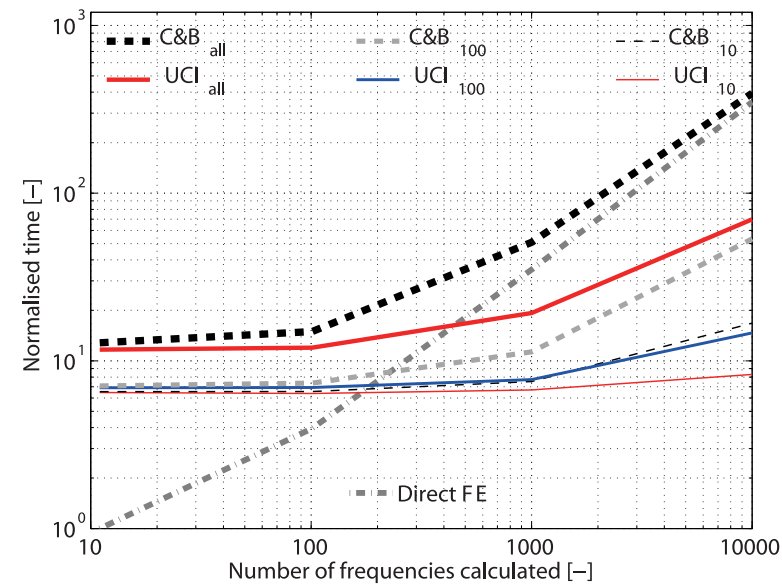

Fig. 11. Normalised time consumption as a function number of frequencies calculated. C\&B method (dashed lines). UCI approach (solid lines) for different number of included inner modes (all, 100 and 10) and the direct solution (dashed-dotted line).

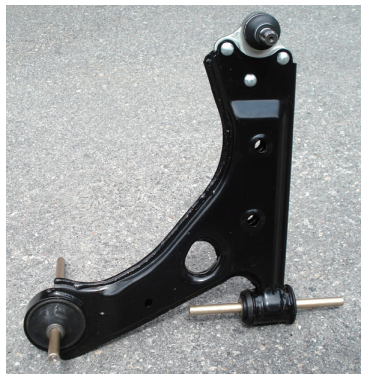

(a)

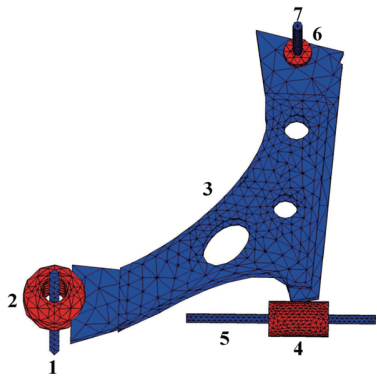

(b)
Fig. 12. Model geometry and inspiration geometry of the linking arm. (a) Inspiration geometry, the linking arm of a vehicle suspension system together with two additional rods; (b) Model geometry, the mesh used to model the linking arm, red parts representing rubber parts, blue part representing steel parts.

neither mesh nor geometry needs to conform. Hence, two different test cases are evaluated in this paper, one simple in which mesh and geometry conform, where C\&B and UCI easily can be compared, and one case with a more complex geometry, in which mesh and geometry do not conform, where the usefulness of the suggested approach is better heightened. The inspiration for the geometry is a so-called linking arm in a vehicle suspension system. The inspiration geometry can be seen in Fig. 12(a). Two rods are added that would not be present in the real suspension system; the rods are added to obtain a more dynamic model without needing to model the entire suspension system. The real geometry consists of several parts including bolts and steel rigs. The model geometry including the mesh can be see in Fig. 12(b); this geometry consists of only seven parts. Hence, bolts and such are not included in the model due to their supposed small influence on the vibro-acoustic response. Nor does the model include as a substructure the steel rigs present in the real geometry in the coupling interfaces of the rubber bushings, but the stiffness effect of these rigs can be argued to be inherently included in the UCI approach since the interfaces are not allowed to deform. The model using the UCI approach in Fig. 12(b) consists of three steel rods (1, 5 and 7) connected to three rubber parts (in red 2, 4 and 6) which are all connected to the boxlike steel linking arm. Since the model only aims to model a geometry with the same type of problems (e.g. connecting parts of fundamentally different stiffness) as the real geometry, part 6 is modelled as a rubber connection although in real geometry there is no such part. The two rods 1 and 5 are modelled with a fixed boundary condition on both ends of the two rods, and rod number 7 has a unit surface force excitation on the free end exciting bending motion of the rod (i.e. no normal force).

The two local coupling interface of each global interfaces are not forced to conform in either size, shape, location or mesh. For example, the two surfaces coupling together part 1 and 2 have different area, the two surfaces have the same dimension in the length direction of the rod but the circumference is different for the two, the surfaces are distant from each other and the mesh is not conforming. The choice of exact properties (size, shape, location and mesh) of each coupling interface is not presented here since this implementation is merely included to show the simplicity of the modelling approach. The steel parts are modelled with a Young's modulus of $200 \mathrm{GPa}$, a mass density of $7850 \mathrm{~kg} / \mathrm{m}^{3}$, a Poisson's ratio of 0.33 and a loss factor of $1 \%$. The rubber is modelled with Young's modulus of $200 \mathrm{kPa}$, a mass density of $1100 \mathrm{~kg} / \mathrm{m}^{3}$, a Poisson's ratio of 0.49 and a loss factor of $10 \%$. Since the purpose of this model is only to show the industrial applicability of the approach, details of the exact geometry modelling condition are left out, and hence, the results should not be viewed as the response of a specific linking arm system.

In Fig. 13 the total translation displacement of the interface connecting parts 1 and 2 is plotted, where the solid line is the solution when keeping all inner modes, and the dashed line is the solution using no inner modes. What can be seen is that below $100 \mathrm{~Hz}$ the responses do not significantly deviate, hence it appears as the clear resonant 


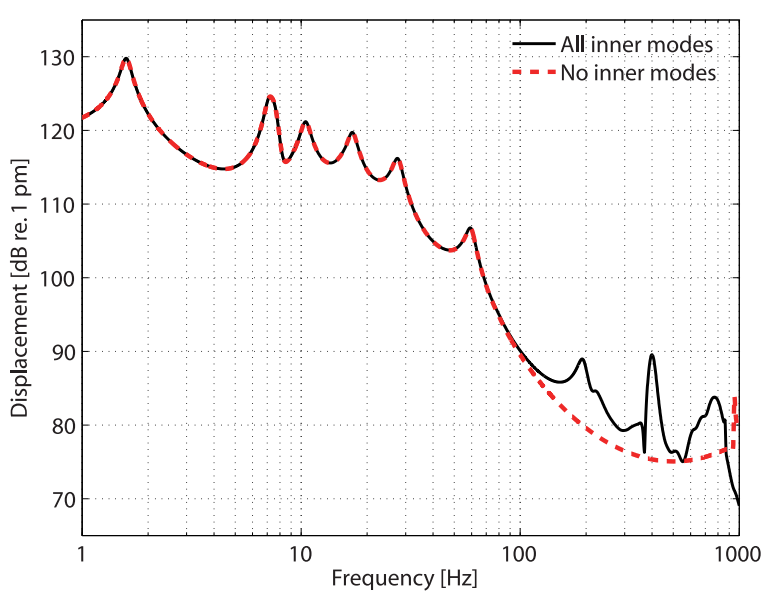

Fig. 13. Vibro-acoustic response (total translation displacement of the coupling interface between part 1 and 2). All inner modes included (solid line) and no inner modes included (dashed line).

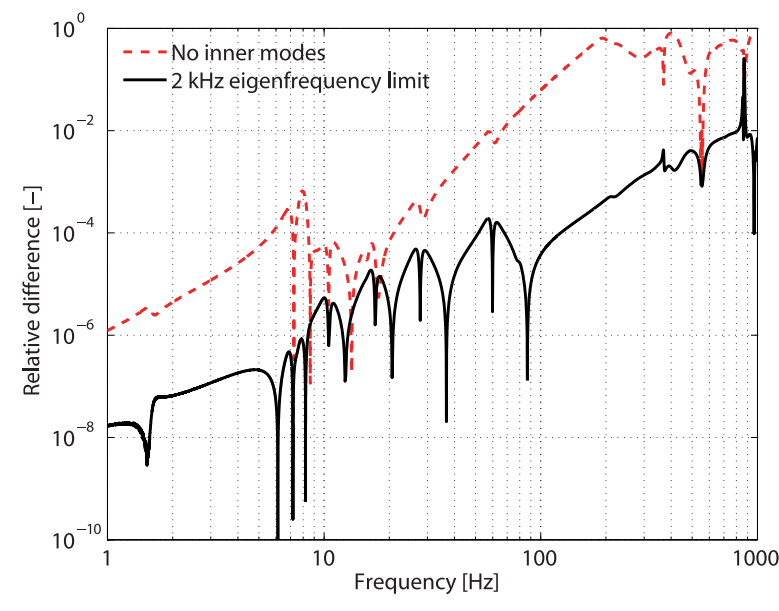

Fig. 14. Relative difference from the direct solution of the solution using no inner modes (dashed line) and the solution using all modes with an eigenfrequency below $2 \mathrm{kHz}$ (solid line), respectively.

behaviour below $100 \mathrm{~Hz}$ may be governed by the mass spring behaviour of the global structure. Above $100 \mathrm{~Hz}$ the resonant behaviour may be govern by the resonant behaviour of the individual components. Figure 14 shows the relative difference between, on the one hand, the direct solution and the modal solution using all inner modes, and, on the other hand, the solution using no inner modes (dashed line) and all local modes with an eigenfrequency below $2 \mathrm{kHz}$ (solid line). It may be noted that below $100 \mathrm{~Hz}$ including mode up to $2 \mathrm{kHz}$ do not majorly enhance the results, over $100 \mathrm{~Hz}$ on the other hand the result gives a engineeringly good representation of the solution using all modes. The total number of DOFs of the original uncoupled problem was 48585 , and the final model using local modes up to $2 \mathrm{kHz}$ modelled the same problem using 1303 DOFs of which 36 is the coupling DOFs. In this paper the soft connections or rubber bushings was merely modelled with frequency independent material properties that in the order of magnitude give the same stiffness properties as rubber would. It is not the aim in this paper to correctly model the rubber; the effective and physical motivated modelling of the rubber bushings are left for a future paper. Hence, the model description of these softer parts is of less importance, and it is interesting to see that the model using the eigenfrequency limit of $2 \mathrm{kHz}$ merely needed a total of seven dynamic inner modes for the steel parts to give an adequate result; for instance, the linking is described by only three local inner modes. The original local FE mesh using a second-order polynomial as basis function is a good representation of the inherent problem of meshing complex geometries. That is, even though the geometry is simplified (from the original CAD) the mesh to describe the geometry is much larger than is needed to describe the vibro-acoustic response.

\section{Conclusions}

A component mode synthesis approach using undeformed coupling interfaces is proposed. The approach enables a significant reduction of the original problems, where classical CMS are limited to reduction of DOFs not associated with the coupling interfaces. The approach also overcomes any problem of the non-conforming mesh of different components.

It is concluded that specific systems can be modelled using the suggested approach, giving results that are valid from an engineering point of view. The stiffness of the connectors is of importance; for the approach to be valid, the stiffness of the connecting bodies must be fundamentally different, as may be the case for the rubber bushing connected to a steel linking arm in the vehicle suspension system.

Finally, the simplicity of the approach is shown for an industrial-like application, namely, a linking arm connected via rubber bushings. 


\section{References}

[1] J.S. Bendat and A.G. Piersol, Engineering applications of correlation and spectral analysis, Wiley, 2nd edition, 1993.

[2] S. Barrett, On the use of coherence functions to evaluate sources of dynamic excitation, Shock and Vibration Bulletin 49 (1979), 43-58.

[3] H. Van der Auweraer, P. Mas, S. Dom, A. Vecchio, K. Janssens and P. Van de Ponseele, Transfer path analysis in the critical path of vehicle refinement: The role of fast, hybrid and operational path analysis, in: SAE Technical Paper series, No. 2007-01-2352.

[4] I. Kido, A. Nakamura, T. Hayashi and A. Makoto, Suspension vibration analysis of road noise using finite elemant model, in: SAE Technical Paper series, No. 1999-01-1788.

[5] R. Craig and M. Bampton, Coupling of substructures for dynamic analyses, AIAA Journal 6(7) (1968), 1313-1319.

[6] D. Rixen, A dual Craig-Bampton method for dynamic substructuring, Journal of Computational and Applied Mathematics 168 (2004), 383-391.

[7] R. MacNeal, A hybrid method of component mode synthesis, Computers and Structures 1 (1971), 581-601.

[8] D. De Klerk, D. Rixen and S. Voormeeren, General framework for dynamic substructuring: History, review, and classification of techniques, AIAA Journal 46 (2008), 1169-1181.

[9] M. Junge, D. Brunner, J. Becker and L. Gaul, Interface-reduction for the Craig-Bampton and Rubin method applied to FE-BE coupling with a large fluid-structure interface, International Journal for Numerical Methods in Engineering 77 (2009), 1731-1752.

[10] D.-M. Tran, Component mode synthesis methods using partial interface modes: Application to tuned and mistuned structures with cyclic symmetry, Computers and Structures 87 (2009), 1141-1153.

[11] E. Balmes, Use of generalized interface degrees of freedom in component mode synthesis, in: Proceedings of the IMAC.

[12] C. Farhat and M. Geradin, A hybrid formulation of a component mode synthesis method, in: AIAA-92-2383.

[13] D. Rixen, C. Farhat and M. Géradin, A two-step, two-field hybrid method for the static and dynamic analysis of substructure problems with conforming and non-conforming interfaces, Computer Methods in Applied Mechanics and Engineering 154 (1998), 229-264.

[14] D.-M. Tran, Component mode synthesis methods using interface modes. Application to structures with cyclic symmetry, Computers and Structures 79 (2001), 209-222.

[15] J. Herrmann, M. Maess and L. Gaul, Substructuring including interface reduction for the efficient vibro-acoustic simulation of fluid-filled piping systems, Mechanical Systems and Signal Processing 24 (2010), 153-163.

[16] K. Gérald, B. Etienne and C. Didier, Model reduction for efficient FEM/BEM coupling, in: Proceedings of ISMA 25 Leuven, Belgium.

[17] E. Balmes, Modes and regular shapes. how to extend component mode synthesis theory, in: Proceedings of the XI DINAME.

[18] P. Van Der Valk, Model reduction and interface modeling in dynamic substructuring, Master's thesis, Delft University of Technology, 2010.

[19] D. De Klerk, Dynamic response characterization of complex systems through operational identification and dynamic substructuring, PhD thesis, Delft University of Technology, 2009.

[20] D. De Klerk, D. Rixen, S. Voormeeren and F. Pasteuning, Solving the RDoF Problem in Experimental Dynamic Substructuring, Proceedings of the Twenty Sixth International Modal Analysis Conference, Orlando, FL, 2008. 

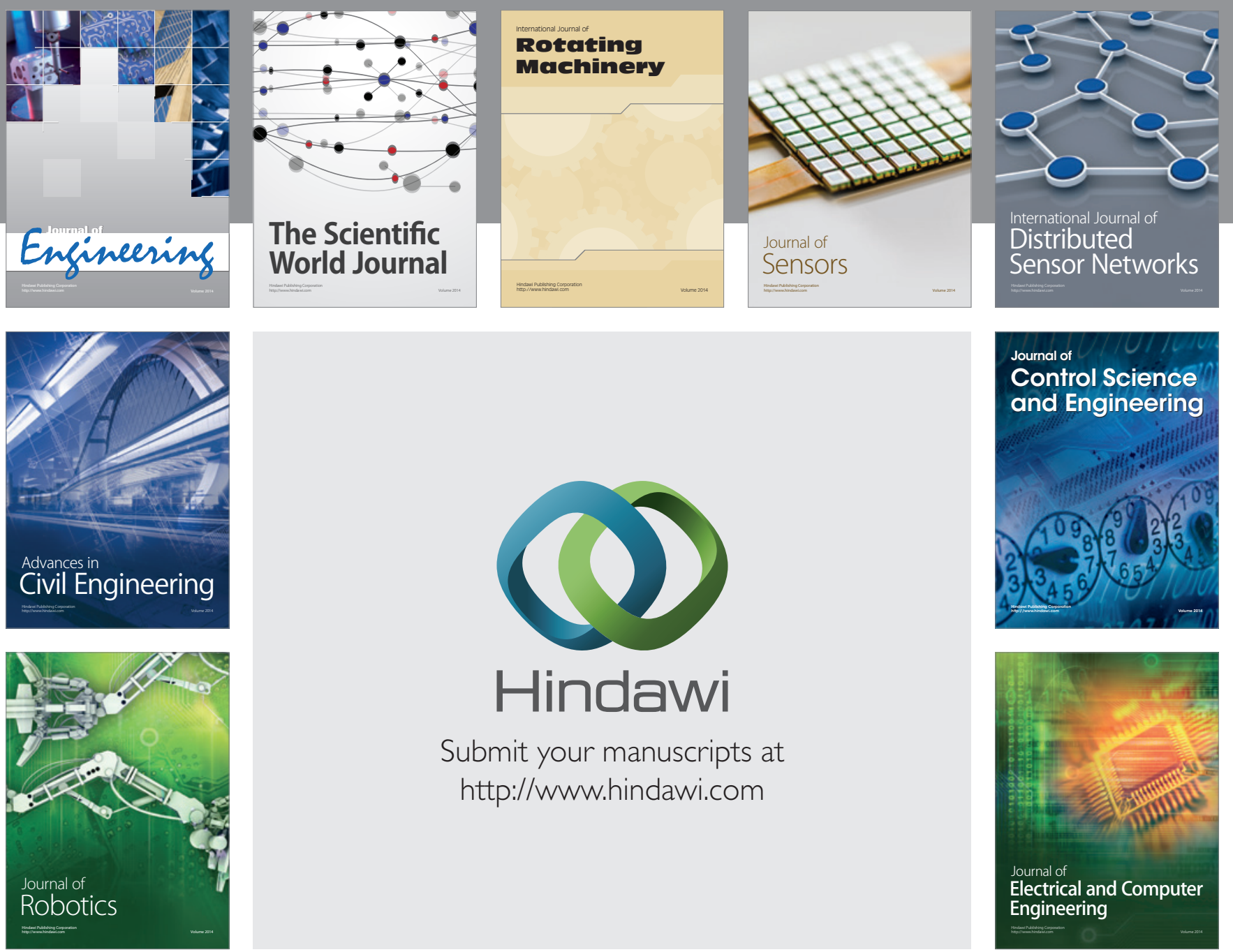

Submit your manuscripts at

http://www.hindawi.com
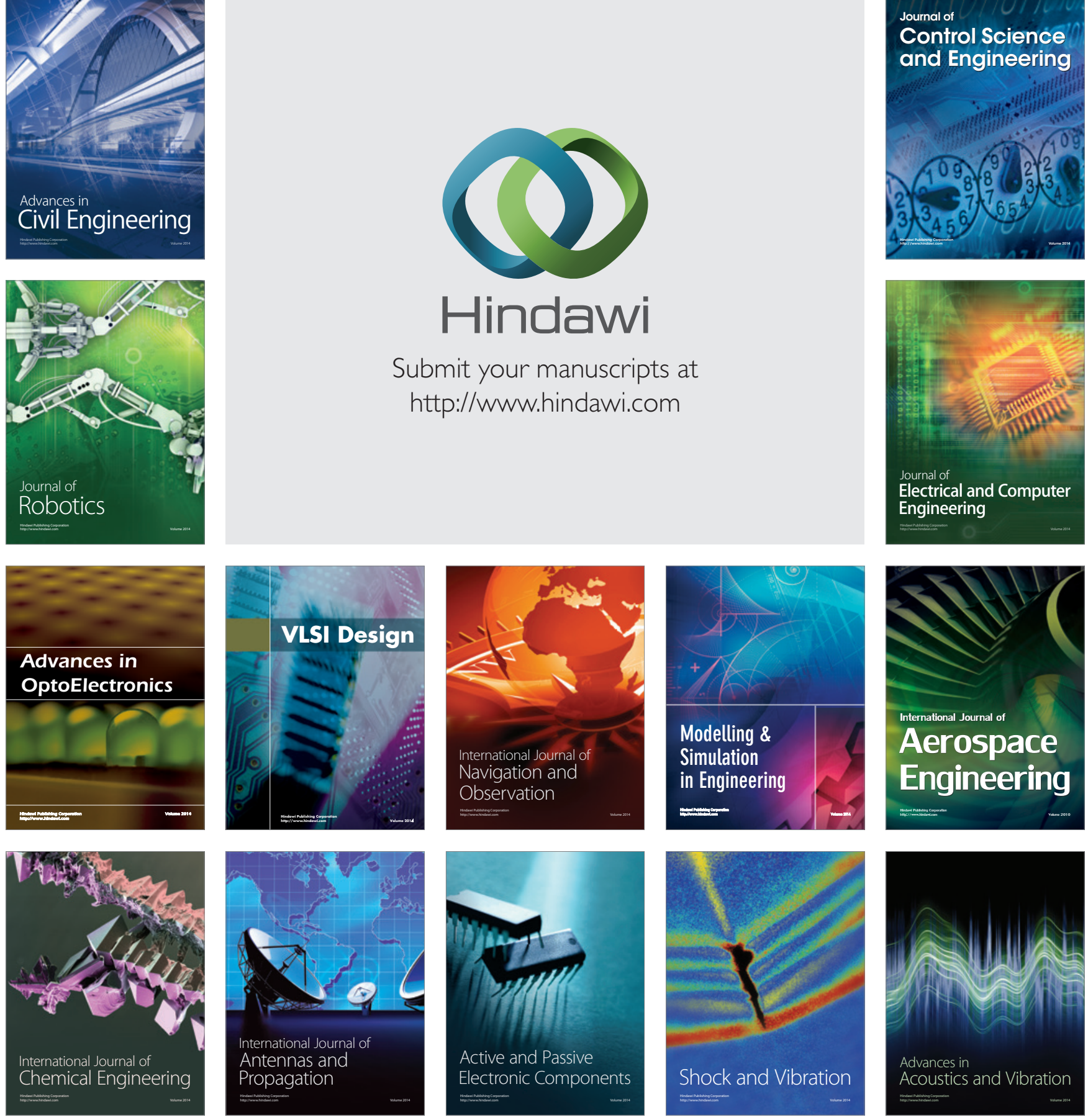